\title{
FICHE INFORMATIVE
}

\section{Portrait de l'entrepreneuriat féminin en contexte de pandémie}

\section{Véronique Dargis ${ }^{\mathrm{a}}$}

L'entrepreneuriat au Québec a connu un véritable essor au cours de la dernière décennie. Les différentes études menées sur le sujet, notamment l'Indice entrepreneurial québécois, témoignent du dynamisme entrepreneurial des Québécois et Québécoises, mais aussi d'une culture entrepreneuriale toujours plus forte. Même constat du côté des femmes, pour qui le taux d'intention d'entreprendre a triplé depuis 2009.

Toutefois, les entreprises à propriété féminine sont encore largement minoritaires au sein des PME québécoises. En effet, seulement 13,6\% des entreprises du Québec sont détenues à $100 \%$ par une ou des femmes, comparativement à 64,9\% d'entreprises détenues par leurs homologues masculins.

De plus, la pandémie est venue toucher durement les entreprises à propriété féminine et celles détenues par des personnes de minorité visible, ce qui nous rappelle la fragilité de nos acquis en matière de parité et d'inclusion.

Au printemps dernier, alors que le Québec venait d'être frappé par la première vague de la pandémie de COVID-19, Femmessor a réalisé une importante enquête, en collaboration avec la Chaire BMO en diversité et gouvernance de l'Université de Montréal et le Portail de connaissances pour les femmes en entrepreneuriat. Au total, ce sont plus de 1025 entrepreneures provenant des 17 régions du Québec qui se sont exprimées sur leurs défis et leurs enjeux.

Deux entreprises à propriété féminine sur trois fonctionnaient alors à moins de $50 \%$ de leurs activités. Parmi les entrepreneures sondées, $42 \%$ étaient également en recherche active de financement pour assurer leur survie ou pour adapter leur offre de services ou produits à la situation. Toutefois, seulement $20 \%$ de ces dernières avaient l'intention de se prévaloir des mesures mises en place par le gouvernement du Québec et par le gouvernement du Canada. Il est fort à parier que cette statistique serait plus élevée aujourd'hui, alors que plusieurs critères d'admissibilité aux différents programmes ont été revus et adaptés. Or, plusieurs entrepreneures ont plutôt dû se tourner vers d'autres sources de financement.

Outre le financement, les entrepreneures ont nommé des moyens de les aider à adapter leur entreprise. Le développement du marketing et du commerce électronique arrive au premier plan. L'élargissement du réseau de contacts et le développement des affaires sont aussi prioritaires. Cela démontre que, pour nos entreprises à propriété féminine, le volet humain est aussi important que le virage numérique.

Finalement, les entrepreneures ont eu l'occasion de s'exprimer sur leurs attentes par rapport à la reprise économique :

- Privilégier l'achat local pour qu'il continue d'être au cœur des priorités;

${ }^{a}$ Directrice du rayonement des affaires 
- Accompagner les entrepreneures pour qu'elles puissent acquérir les compétences nécessaires à la croissance de leur entreprise (numérique et gestion financière);

- Faire évoluer le modèle économique global, notamment en ce qui a trait à l'importance de préserver l'environnement et de favoriser les mesures de conciliation travail-famille, dont le télétravail.

Bref, ce portrait démontre la grande fragilité actuelle des entreprises à propriété féminine. En même temps, on a pu constater la résilience des entrepreneures, qui travaillent ardemment pour survivre et pour faire pivoter leur entreprise afin d'être prêtes lors de la relance économique. Femmessor a d'ailleurs adapté son offre de services afin de mieux accompagner les entrepreneures du Québec dans cette perspective.

Pour en savoir plus sur Femmessor : www.femmessor.com 